\title{
Gestión educativa como factor determinante del desempeño de docentes de educación básica regular durante la pandemia COVID-19, Puno-Perú
}

\author{
Educative management as a determining factor in the performance of regular basic \\ education teachers during the COVID-19 pandemic, Puno-Peru.
}

\author{
Lucas Fernando Meza Revatta ${ }^{1}$, Julio Samuel Torres Miranda ${ }^{2}$ y Oscar Mamani-Benito ${ }^{3 a}$
}

Escuela profesional de Administración, Universidad Peruana Unión Juliaca, Perú ${ }^{123}$

(iD https://orcid.org/0000-0003-3702-9766

(iD https://orcid.org/0000-0002-9818-26012

(iD) https://orcid.org/0000-0001-5951-8849

Recibido: 12 de junio de 2020

Aceptado: 15 de setiembre de 2020

\begin{abstract}
Resumen
La presente investigación tuvo como objetivo analizar el efecto de la gestión educativa sobre el desempeño docente en profesores de Educación Básica Regular del departamento de Puno, durante la Pandemia COVID-19. Corresponde a una investigación de enfoque cuantitativo, alcance explicativo y diseño no experimental, que contó con la participación de 650 docentes de 10 Unidades de Gestión Educativa Local, a quienes se les aplicó una escala de percepción de la gestión educativa y un cuestionario de autoevaluación del desempeño docente, ambos válidos y confiables; los análisis se realizaron en base a un nivel de significancia del $5 \%$ y nivel de confianza del $95 \%$. Los resultados dan cuenta de una correlación directa y significativa entre la variable gestión educativa y desempeño docente $(\mathrm{r}=.623, \mathrm{p}<.05)$, de igual forma con las dimensiones gestión institucional $(\mathrm{r}=.523, \mathrm{p}<.05)$, Gestión pedagógica $(\mathrm{r}=.499, \mathrm{p}<0.05)$, Gestión administrativa $(\mathrm{r}=.611, \mathrm{p}<.05)$ y Gestión comunitaria $(\mathrm{r}=.514, \mathrm{p}<.05)$. Aunado a esto, el modelo de regresión lineal múltiple explica el efecto que tiene la variable gestión educativa y sus dimensiones, sobre el desempeño docente en los profesores de Puno. Se concluye que la gestión educativa, que puede ser entendida como el fortalecimiento de los Proyectos Educativos y el enriquecimiento de los procesos pedagógicos con el fin de responder a las necesidades educativas locales y regionales, es un factor determinante que explica el nivel de desempeño que logran los profesores de Educación Básica Regular de Puno durante la Pandemia COVID-19.
\end{abstract}

Palabras claves: gestión educativa, desempeño docente, calidad educativa, educación, covid19

${ }^{\mathrm{a} C}$ Correspondencia al autor:

E-mail: oscar.mb@upeu.edu.pe 


\begin{abstract}
The objective of this research was to analyze the effect of educational management on the teaching performance of teachers of Basic Basic Education in the department of Puno, during the COVID-19 Pandemic. It corresponds to an investigation with a quantitative approach, explanatory scope and non-experimental design, which included the participation of 650 teachers from 10 Units of Local Educational Management, to whom a scale of perception of educational management and a self-assessment questionnaire of the teaching performance, both valid and reliable; The analyzes were performed based on a significance level of $5 \%$ and a confidence level of $95 \%$. The results show a direct and significant correlation between the variable educational management and teacher performance $(\mathrm{r}=.623, \mathrm{p}<.05)$, in the same way with the institutional management dimensions $(\mathrm{r}=.523, \mathrm{p}<.05)$, Pedagogical management $(\mathrm{r}=$ $.499, \mathrm{p}<0.05)$, Administrative management $(\mathrm{r}=.611, \mathrm{p}<.05)$ and Community management $(\mathrm{r}$ $=.514, \mathrm{p}<.05)$. In addition to this, the multiple linear regression model explains the effect that the educational management variable and its dimensions have on the teaching performance of teachers in Puno. It is concluded that educational management, which can be understood as the strengthening of Educational Projects and the enrichment of pedagogical processes in order to respond to local and regional educational needs, is a determining factor that explains the level of performance they achieve teachers of Regular Basic Education in Puno during the COVID19 Pandemic.
\end{abstract}

Keywords: educational management, teacher performance, educational quality, education, covid19

\title{
Introducción
}

La llegada del COVID-19 a Latinoamérica viene generando un impacto negativo principalmente en el ámbito de la salud pública, por lo que casi todos los países han optado por declarar estado de emergencia, aislamiento social y cuarentenas para mitigar las consecuencias (Alvarez \& Harris, 2020). Al mismo tiempo, uno de los rubros también afectados ha sido la educación donde no solo los estudiantes tienen que enfrentar el reto de regular su aprendizaje vía online, sino que los profesores, y sobre todo quienes están en la parte administrativa tienen el reto de gestionar y adaptar el nuevo proceso de enseñanza-aprendizaje (Britez, 2020).

En el Perú, el impacto del COVID-19 se suma a las demandas educativas actuales de este país. A pesar de haber determinado en un consenso un nuevo rumbo para la política educativa peruana expresado en el Proyecto Educativo Nacional (PEN) al 2021 (Ministerio de Educación, 2007), la gestión educativa en las instituciones a escala nacional aún no ha mejorado, persisten los problemas en los diferentes aspectos de las gestiones institucional, pedagógica, administrativa y comunitaria (El Comercio, 2019).

En tal sentido, desde hace varios años, el Ministerio de Educación ha desarrollado instrumentos para mejorar el desempeño docente en instituciones educativas públicas y 
privadas del estado peruano, esta iniciativa se denominó "Marco de Buen Desempeño Docente" (Ministerio de Educación, 2012), el mismo, constituye una herramienta que indica los dominios, competencias y desempeño que todo profesor de educación básica regular debe poseer para evidenciar su buen desempeño como docente. Asimismo, este documento indica que "los cuestionamientos sociales a los sistemas escolares y a los propios docentes exigen sistemas de desarrollo profesional que aseguren una formación del magisterio a la altura de los cambios sociales, que los acompañe y hasta los anticipe" (Ministerio de Educación, 2012, p.6).

Ante tal escenario, se comenta y se exige mucho sobre la gestión educativa y el buen desempeño que cada docente debe ejercer en sus aulas (Gálvez \& Milla, 2018), no obstante, la realidad demuestra que el alcance de este desempeño se ha desvalorizado justamente por cuestiones administrativas y la poca inversión en el activo personal docente (Peru 21, 2019). Por ello, en el contexto internacional, la situación de la educación básica en el Perú no era alentadora, aunque en los resultados de la última evaluación del programa internacional para la Evaluación de Estudiantes (PISA) (La Republica, 2019), Perú subió al puesto 64 de 77 países, subiendo en puntajes de lectura, matemática y ciencia; sin embargo, según la opinión de expertos esta este país aún tiene que enfrentar muchos retos en el siglo XXI (Rojas, 2015).

Por otro lado, analizando el proceso educativo desde el papel de los docentes articulado a las condiciones de la gestión educativa en la que se desempeñan, este viene a ser otro de los factores ineludibles que determina la calidad de la educación; en este entender, Torres (2015) afirma que es ineludible el hecho de que la calidad de la práctica docente, está ligado a la capacidad de gestión educativa por parte de los administradores de turno; y por el lado de los docentes también es necesario enfatizar el hecho de que en la mayoría de ciudades del Perú y algunos países de Latinoamérica se observan dificultades como el poco interés para una capacitación continua, dificultad en el manejo adecuado del salón o aula de clases, dificultad en el manejo del enfoque por competencias, poco compromiso para el alcance de los objetivos institucionales, doble jornada laboral y lo que aún es más vital falta de estímulos o incentivos (Rodríguez-Sosa \& Hernández-Sánchez, 2018; Tapia \& Tipula, 2017).

Si estas dificultades continúan, las instituciones educativas podrían verse afectadas en asuntos de carácter administrativo y de gestión educativa, en general, afectando de manera negativa a su agente principal: el estudiante. Al respecto, la realidad en el departamento de Puno también revela las deficiencias mencionadas en párrafos anteriores, por ejemplo, en un informe reciente realizado por el Ministerio de Educación (2017) a través de la Unidad Estadística se 
enfatiza que aunque este departamento ha mejorado en cuestión económica y social, aun se observa una brecha en cuestión de acceso y calidad de educación, sobre todo en ámbitos rurales.

Ante ello, para que se cumplan todas las dimensiones: gestión institucional, gestión pedagógica, gestión administrativa y gestión comunitaria; de forma productiva es necesario el análisis de la variable gestión educativa como factor que determina el buen desempeño docente, esto, con el fin de plantear soluciones adecuadas y oportunas en beneficio de la formación de los estudiantes. Por todo lo dicho anteriormente, es que el objetivo analizar el efecto de la gestión educativa sobre el desempeño docente en profesores de Educación Básica Regular del departamento de Puno, durante la Pandemia COVID-19.

\section{Método}

El diseño de este estudio es explicativo de diseño no experimental y corte transversal (Hernandez et al., 2014). Los participantes consistieron en una muestra de 650 profesores de ambos sexos de Educación Básica Regular (inicial, primaria y secundaria) que pertenecen a 10 Unidades de Gestión Educativa Local (UGEL) del departamento de Puno: Azángaro (6.92\%), El Collao (9.23\%), Melgar (8.62\%), Puno (19.23\%), San Román (15.08\%), Huancané (7.08\%), Lampa (8.62\%), Yunguyo (11.69\%), Carabaya (6\%) y Chucuito (7.54\%).

\section{Tabla 1}

Distribución de la muestra de estudio según UGEL de procedencia

\begin{tabular}{lcc} 
Unidad de Gestión Educativa Local & $\mathrm{f}$ & $\%$ \\
\hline Ugel Azangaro & 45 & 6.92 \\
Ugel El Collao & 60 & 9.23 \\
Ugel Melgar & 56 & 8.62 \\
Ugel Puno & 125 & 19.23 \\
Ugel San Roman & 98 & 15.08 \\
Ugel Huancané & 46 & 7.08 \\
Ugel Lampa & 56 & 8.62 \\
Ugel Yunguyo & 76 & 11.69 \\
Ugel Carabaya & 39 & 6.0 \\
Ugel Chucuito & 49 & 7.54 \\
Total & 650 & 100.0 \\
\hline
\end{tabular}


Todos los participantes no evidenciaron posibles condiciones físicas o psicológicas que imposibilite la participación en la investigación.

\section{Instrumentos}

En cuanto al desempeño docente: se utilizó un cuestionario adaptado por Reyes (2012) en una investigación similar. El objetivo de la misma es evaluar el grado de desempeño de las funciones del profesor desde la perspectiva del propio docente. Esta prueba consta de 45 ítems distribuidos en cuatro dimensiones, teniendo como opciones de respuesta niveles tipo Lickert: nunca, casi nunca, a veces, casi siempre y siempre. En cuanto a sus propiedades psicométricas, este fue sometido juicio de expertos, quienes ponderaron sus evaluaciones obteniendo un coeficiente V de Aiken de .90; asimismo, se halló un nivel de consistencia interna mediante el Alpha de Cronbach de .911. Lo cual indica que es totalmente valido y confiable. En cuanto a la gestión educativa: se utilizó una escala elaborada por Pérez (2010) en una investigación anterior, y adaptada para la población de la presente investigación. Tiene el objetivo de evaluar el nivel de gestión educativa que realizan los directivos en base a la percepción de los profesores. Consta de 40 ítems distribuidos en cuatro dimensiones, con opciones de respuesta tipo Lickert: nunca, casi nunca, a veces, casi siempre y siempre. En cuanto a sus propiedades psicométricas, este fue sometido juicio de expertos, quienes ponderaron sus evaluaciones obteniendo un coeficiente V de Aiken de .95; así mismo se halló un nivel de consistencia interna mediante el Alpha de Cronbach de .916. Lo cual indica que es totalmente valido y confiable.

La recolección de datos fue realizada entre los meses de Marzo -Abril de 2020. Antes de aplicar las encuestas se gestionó la autorización de la Unidad de Gestión Educativa del departamento de Puno, de igual modo, se coordinó las fechas de aplicación. En una primera etapa se aplicó una encuesta documental presencial, mientras que en la segunda etapa se continuó con encuestas online mediante formularios de google debido al aislamiento social obligatorio por el COVID-19. Posteriormente se hizo control de calidad de la data en MS Excel.

\section{Análisis estadístico}

Para el análisis estadístico se utilizó el programa SPSS versión 22.0. Los resultados descriptivos se expresaron en tablas de frecuencia y porcentaje, en cambio, el análisis inferencial se generó en base a un nivel de significancia del $5 \%(\mathrm{p}=.05)$. Por otra parte, el estadístico de prueba fue elegido en razón de la prueba de normalidad ( $\mathrm{K}-\mathrm{S}>30)$, con lo cual, se logró determinar cómo apropiado una prueba paramétrica, en este caso, $\mathrm{r}$ de Pearson. Finalmente, para evaluar el efecto de la variable independiente sobre la variable dependiente se realizó un análisis de regresión lineal múltiple. 
El estudio respeta la ética en la investigación. Evidencia de ello, es que se procedió a gestionar a la autorización por la UGEL, así mismo, se obtuvo el consentimiento informado de los participantes tanto en la fase presencial como en la fase virtual. Se guardó total confidencialidad respecto a los datos brindados.

\section{Resultados}

Según la tabla 2, se observa en mayor proporción que el 37.23\% de participantes tiene entre 28 a 35 años de edad, seguido por un 24\% que tiene entre 36 a 45 años, $22.31 \%$ entre 21 a 27 años, y en menor proporción un $16.46 \%$ entre 26 a 55 años. Por otra parte, $61.23 \%$ son mujeres y $38.70 \%$ varones. En cuanto a la religión que profesan, $64.77 \%$ son católicos, $19.08 \%$ son evangélicos y $9.23 \%$ adventistas. En otro aspecto, $53.23 \%$ enseñan en el nivel secundario, $32.31 \%$ en el nivel primario y $13.85 \%$ en el nivel inicial.

\section{Tabla 2}

Características demográficas de la población de estudio

\begin{tabular}{lccc} 
Variable & Categoría & Frecuencia & Porcentaje \\
\hline Edad & $21-27$ años & 145 & 22.31 \\
& $28-35$ años & 242 & 37.23 \\
& $36-45$ años & 156 & 24.00 \\
& $46-55$ años & 107 & 16.46 \\
Sexo & Total & 650 & 100.0 \\
& Masculino & 252 & 38.70 \\
Religión & Femenino & 398 & 61.23 \\
& Total & 650 & 100.0 \\
& Católico & 421 & 64.77 \\
& Evangélico & 124 & 19.08 \\
& Adventista & 60 & 9.23 \\
Nivel & Otros & 45 & 6.92 \\
& Total & 650 & 100.0 \\
& Inicial & 94 & 13.85 \\
& Primaria & 210 & 32.31 \\
& Secundaria & 346 & 53.23 \\
& Total & 650 & 100.0 \\
\hline
\end{tabular}

En la tabla 3, se observa los niveles de gestión educativa y desempeño docente desde la percepción de los profesores. Así, el 58.7\% revela un nivel regular de desempeño docente, un $27.4 \%$ un nivel bueno y un $14.2 \%$ un nivel malo. En cuanto a la gestión educativa, $53.8 \%$ opina que se realiza una mala gestión, 32.8\% de manera regular y solo un 13.4\% una buena gestión. En la misma línea, $64.2 \%$ opina que la gestión institucional es mala y solo un $12.2 \%$ buena, el 
resto opina que es regular; una realidad similar de alto porcentaje de desaprobación recibe gestión pedagógica (64.5\%), gestión administrativa (70.2\%); mientras que en gestión comunitaria $63.5 \%$ refiere ser regular.

\section{Tabla 3}

Análisis descriptivo de las variables de estudio

\begin{tabular}{|c|c|c|c|}
\hline Variable & Categoría & $\mathrm{F}$ & $\%$ \\
\hline \multirow{4}{*}{ Desempeño docente } & Malo & 92 & 14.2 \\
\hline & Regular & 380 & 58.5 \\
\hline & Bueno & 178 & 27.4 \\
\hline & Total & 650 & 100.0 \\
\hline \multirow[t]{4}{*}{ Gestión educativa } & Malo & 350 & 53.8 \\
\hline & Regular & 213 & 32.8 \\
\hline & Bueno & 87 & 13.4 \\
\hline & Total & 650 & 100.0 \\
\hline \multirow[t]{4}{*}{ Gestión institucional } & Malo & 417 & 64.2 \\
\hline & Regular & 154 & 23.7 \\
\hline & Bueno & 79 & 12.2 \\
\hline & Total & 650 & 100.0 \\
\hline \multirow[t]{4}{*}{ Gestión pedagógica } & Malo & 419 & 64.5 \\
\hline & Regular & 178 & 27.4 \\
\hline & Bueno & 53 & 8.2 \\
\hline & Total & 650 & 100.0 \\
\hline \multirow[t]{4}{*}{ Gestión administrativa } & Malo & 456 & 70.2 \\
\hline & Regular & 120 & 18.5 \\
\hline & Bueno & 74 & 11.4 \\
\hline & Total & 650 & 100.0 \\
\hline \multirow[t]{4}{*}{ Gestión comunitaria } & Malo & 167 & 25.7 \\
\hline & Regular & 413 & 63.5 \\
\hline & Bueno & 70 & 10.8 \\
\hline & Total & 650 & 100.0 \\
\hline
\end{tabular}

Según la tabla 4, el análisis de normalidad según el estadístico Kolmogorov-Smirnov revela que los valores de sig. en todos los casos superan el 0.05 , por lo tanto, se acepta la hipótesis nula que implica que los datos siguen una distribución normal. 


\section{Tabla 4}

Análisis de normalidad

\begin{tabular}{lccc} 
& \multicolumn{3}{c}{ Kolmogorov-Smirnov } \\
& Estadístico & Gl & Sig. \\
\hline Desempeño docente & 0.078 & 85 & 0.15 \\
Gestión docente & 0.056 & 85 & 0.23 \\
Gestión institucional & 0.089 & 85 & 0.06 \\
Gestión pedagógico & 0.084 & 85 & 0.18 \\
Gestión administrativo & 0.078 & 85 & 0.21 \\
Gestión comunitaria & 0.086 & 85 & 0.07 \\
\hline
\end{tabular}

En la tabla 5 se observa la existencia de correlaciones significativas; tal es el caso de gestión educativa y desempeño docente con un nivel de relación alto, directo y significativo (r $=.623, \mathrm{p}<.05)$. De igual forma, una relación moderada, directa y significativa con gestión institucional $(\mathrm{r}=.523, \mathrm{p}<.05)$, una relación directa, de magnitud moderada y significativa con gestión pedagógica $(\mathrm{r}=.499, \mathrm{p}<.05)$, una relación directa, de magnitud alta y significativa con la gestión administrativa $(\mathrm{r}=.611, \mathrm{p}<.05)$; finalmente, una relación directa, moderada y significativa con la gestión comunitaria $(\mathrm{r}=.514, \mathrm{p}<.05)$.

\section{Tabla 5}

Análisis de correlación entre las variables de estudio

Desempeño docente

\begin{tabular}{lcc} 
Variable independiente & $\mathrm{r}$ & $\mathrm{P}$ \\
\hline Gestión educativa & $.623^{* *}$ & .002 \\
Gestión institucional & $.523^{* *}$ & .001 \\
Gestión pedagógico & $.499^{* *}$ & .000 \\
Gestión administrativo & $.611^{* *}$ & .002 \\
Gestión comunitaria & $.514^{*}$ & .031 \\
\hline
\end{tabular}

En la Tabla 6 se observa que el modelo de regresión lineal múltiple explica el efecto de los indicadores de la variable gestión educativa sobre el desempeño docente. Reconociendo que los indicadores son cuantitativos, luego de contrastar los supuestos estadísticos para validar el modelo, los hallazgos demuestran que las cuatro dimensiones: gestión institucional, administrativa, pedagógica y comunitaria, explican la variabilidad del desempeño docente en un 52.4\%. Finalmente, según el resultado de la prueba t de Student, se asume que la dimensión gestión administrativa es el predictor más importante $(\mathrm{t}=2.971, \mathrm{p}<.05)$, seguido por gestión 
institucional $(\mathrm{t}=2.167, \mathrm{p}<.05)$, gestión pedagógica $(\mathrm{t}=2.156, \mathrm{p}<.05)$ y gestión comunitaria $(\mathrm{t}=2.145, \mathrm{p}<.05)$.

\section{Tabla 6}

Coeficientes de regresión lineal múltiple en base al efecto de los indicadores de la gestión educativa sobre el desempeño docente

\begin{tabular}{lrccc} 
Modelo: & \multicolumn{2}{c}{ Coeficientes no estandarizados } & & \\
$\mathrm{R}^{2}$ ajustado $=.524$ & $\mathrm{~B}$ & Error estandar & $\mathrm{t}$ de student & $\mathrm{p}$ \\
\hline Constante & 54.672 & 3.251 & 21.768 & 0.00 \\
Gestión institucional & 0.561 & 0.213 & 2.167 & 0.00 \\
Gestión pedagógico & 0.451 & 0.241 & 2.156 & 0.00 \\
Gestión administrativo & 0.651 & 0.189 & 2.971 & 0.00 \\
Gestión comunitaria & 0.461 & 0.176 & 2.145 & 0.03 \\
\hline
\end{tabular}

Variable dependiente: desempeño docente

\section{Discusión}

Se tuvo como objetivo analizar el efecto de la gestión educativa sobre el desempeño docente en profesores de Educación Básica Regular de Puno. Los resultados brindan evidencia de la importancia de optimizar la gestión educativa para impulsar un desempeño adecuado en los docentes en pos de mejorar la calidad educativa, esto en función del trabajo en equipo, la calidad de los procesos y el ejercicio del liderazgo directivo.

En relación a la hipótesis general, los resultados indican una correlación directa y significativa, lo que quiere decir que a mejor gestión educativa mayor desempeño docente se genera en los profesores de educación básica regular. Estos resultados están acorde a los encontrados por otra investigación realizada en el departamento de Puno en el año 2014, en este caso se estudió a profesores de instituciones emblemáticas (Torres, 2015). Ante ello, una explicación teórica surge al comprender la importancia del rol que cumplen quienes se hacen de cargo de la administración educativa en determinados contextos, pues todos los esfuerzos realizados en la gestión deberían estar orientados a la generación de calidad educativa, y esto, únicamente se logrará si se orienta y conduce la labor docente y administrativa de la escuela y sus relaciones con el entorno (Graffe, 2002), entonces, dirigir una organización en este contexto, implica que las acciones, estrategias, políticas y decisiones que se toman a nivel administrativo sean para la mejora educativa de manera continua (Inciarte et al., 2006), donde los profesores son pieza clave pues actúan como mediadores para el proceso-aprendizaje de los 
estudiantes, y el cumplimiento adecuado de sus funciones dependerá de los recursos, estrategias, incentivos que puedan percibir por parte de sus superiores (Aguerrondo, 2014).

En relación a las hipótesis específicas, se hallaron correlaciones directas y significativas entre las dimensiones de gestión educativa y la variable desempeño docente, además, el análisis de regresión indica que Gestión institucional, Gestión pedagógica, Gestión administrativa y Gestión comunitaria explican en un 52.4\% la variabilidad del desempeño docente. Resultados similares fueron hallados en otras investigaciones, por ejemplo, Quispe-Pareja (2020) estudió la relación entre la gestión pedagógica y el desempeño docente en profesores del cercado de Lima, hallando una correlación positiva de magnitud moderada; por otra parte, Surco (2018) investigó la relación entre la gestión académica y el desempeño docente en una universidad privada de Lima, hallando también un relación positiva. Ahora, en cuanto a la gestión administrativa, Pacheco-Granados et al. (2018) analizaron la situación administrativa educativa rural en Santa Martha, Colombia, un contexto similar al de Puno, concluyendo que no se evidenció un enfoque administrativo predominante y que existe moderada presencia en el uso de técnicas de investigación operacional en la gestión administrativa de los colegios. Esta situación es también la que revelan los docentes del departamento de Puno, pues más del 50\% calificó como mala la gestión educativa que realizan los actuales administradores en la Dirección Regional de Educación y las Unidades de Gestión Educativa Local.

Las implicancias de estos resultados orientar a tomar conciencia respecto a la administración educativa que actualmente se ejerce en el departamento de Puno, que desde hace muchos años viene luchando por no ser más una de las regiones más pobres del Perú (León, 2019), aunado a esto, la búsqueda de una educación inclusiva debido a que gran parte de la población estudiantil corresponde al ámbito rural (Huanca-Arohuanca \& Canaza-Choque, 2019). Por todo ello y ante los hallazgos de la presente investigación se hace urgente fortalecer la gestión educativa en esta parte del Perú, donde los administradores tienen una tarea vital, para lo cual, necesitan prepararse como tales, puesto que son ellos los gestores de una educación de calidad (Garcia et al., 2018).

En conclusión, la gestión educativa, a través de las acciones y estrategias que permiten dirigir los recursos humanos, materiales y financieros del sector educación (gestión administrativa), el marco de referencia para orientar la planificación, programación y evaluación del quehacer institucional (gestión institucional), el liderazgo y control que ejecuta el personal directivo de para hacer cumplir la normativa sobre el desarrollo de las actividades 
(gestión pedagógica) y los mecanismos orientados al análisis de las necesidades y capacidad de respuesta para la sociedad (gestión comunitaria), predicen el nivel de cumplimiento óptimo de funciones y rendimiento profesional del docente de educación básica regular, en este caso del departamento de Puno.

\section{Referencias}

Aguerrondo, I. (2014). Planejamento Educativo E Complexidade: Gestão Das Reformas Educacionais. Cadernos de Pesquisa, 44(153), 548-578. https://doi.org/10.1590/198053142910

Alvarez, R. P., \& Harris, P. R. (2020). Covid-19 in latin america: Challenges and opportunities. Revista Chilena de Pediatria, 91(2), 179-182. https://doi.org/10.32641/rchped.vi91i2.2157

Britez, M. (2020). La educación ante el avance del COVID-19 en Paraguay . Scientific Electronic Library Online. https://preprints.scielo.org/index.php/scielo/preprint/view/22/579

El Comercio. (2019). Educación en el Perú: Calidad educativa no estaría justificando las inversiones. Recuperado de https://elcomercio.pe/economia/peru/educacion-peru-calidadeducativa-estaria-justificando-inversiones-noticia-619726-noticia/

Gálvez, E., \& Milla, R. (2018). Evaluación del desempeño docente: Preparación para el aprendizaje de los estudiantes en el Marco de Buen Desempeño Docente. Propósitos y Representaciones, 6(2), 407. https://doi.org/10.20511/pyr2018.v6n2.236

Garcia, F., Juarez, S., \& Salgado, L. (2018). Gestión escolar y calidad educativa S. Revista Cubana Educación Superior, 2(6), 206-216. Recuperado de http://scielo.sld.cu/pdf/rces/v37n2/rces16218.pdf

Graffe, G. (2002). Gestión educativa para la transformación de la escuela. Revista de Pedagogia, 23(68), 12-21. Recuperado de http://ve.scielo.org/scielo.php?script=sci_arttext\&pid=S0798-97922002000300007

Hernandez, R., Fernández, C., \& Baptista, P. (2014). Metodología de la investigación (6th ed.). McGrawHill.

Huanca-Arohuanca, J., \& Canaza-Choque, F. (2019). Puno: Educación rural y pensamiento crítico. Hacia una educación inclusiva. Revista Helios, 3(1), 2019. 
https://doi.org/10.22497/Helios.31.3106

Inciarte, A., Marcano, N., \& Reyes, M. (2006). Gestión académico-administrativa en la educación básica. Revista Venezolana De Gerencia, 11(34), 34-47. Recuperado de http://ve.scielo.org/scielo.php?script=sci_arttext\&pid=S1315-99842006000200005

La Republica. (2019). Prueba Pisa: Perú se ubica en el puesto 64 y sube puntaje en lectura, matemática y ciencia. Recuperado de https://larepublica.pe/sociedad/2019/12/03/pruebapisa-peru-se-ubica-en-el-puesto-64-y-sube-puntaje-en-lectura-matematica-y-cienciaminedu-educacion/

León, J. C. (2019). Capital humano y pobreza regional en Perú. Región Y Sociedad, 31(1), e1058. https://doi.org/10.22198/rys2019/31/1058

Ministerio de Educación. (2007). Proyecto Educativo Nacional PEN. Recuperado de http://www.minedu.gob.pe/DeInteres/xtras/PEN-2021.pdf

Ministerio de Educación. (2012). Marco de Buen Desempeño Docente. Recuperado de http://www.minedu.gob.pe/pdf/ed/marco-de-buen-desempeno-docente.pdf

Ministerio de Educación. (2017). Puno ¿cómo vamos en educación? Unidad de Estadística. Recuperado de http://escale.minedu.gob.pe/c/document_library/get_file?uuid=4e9029ea5a7d-4e76-96c7-42acaa92ec4f\&groupId=10156

Pacheco-Granados, R. J., Robles-Algarín, C. A., \& Ospino-Castro, A. J. (2018). Analysis of the administrative management in the educational institutions of the basic and middle levels in the rural areas of Santa Marta, Colombia. Informacion Tecnologica, 29(5), 259-266. https://doi.org/10.4067/S0718-07642018000500259

Perez, J. (2010). Administración y Gestión Educativa desde la perspectiva de las Practicas de Liderazgo y el ejercicio de los Derechos Humanos en la Escuela Normal Mixta "Pedro Nufio" [Universidad Pedagogica Nacional Francisco Morazán]. http://www.cervantesvirtual.com/descargaPdf/administracion-y-gestion-educativa-desdela-perspectiva-de-las-practicas-de-liderazgo-y-el-ejercicio-de-los-derechos-humanos-enla-escuela-nomal-mixta-pedro-nufio/

Peru 21. (2019). ¿Cuál es la real inversión en educación en el Perú? Recuperado de https://peru21.pe/opinion/real-inversion-educacion-peru-465792-noticia/

Quispe-Pareja, M. (2020). La gestión pedagógica en la mejora del desempeño docente. Investigación Valdizana, 14(1), 7-14. Recuperado de 
http://revistas.unheval.edu.pe/index.php/riv/article/view/601

Reyes, N. (2012). Liderazgo directivo y desempeño docente en el nivel secundario de una institución educativa de Ventanilla - Callao [Universidad San Ignacio de Loyola]. Recuperado de http://repositorio.usil.edu.pe/bitstream/123456789/1302/1/2012_Reyes_Liderazgo directivo y desempeño docente en el nivel secundario de una institución educativa de Ventanilla- Callao.pdf

Rodríguez-Sosa, J., \& Hernández-Sánchez, K. (2018). Problematización de las prácticas docentes y contextualización de la enseñanza. Propósitos y Representaciones, 6(1), 507524. https://doi.org/10.20511/pyr2018.v6n1.211

Rojas, A. M. (2015). Retos a la Educación Peruana en el Siglo XXI. REICE. Revista Iberoamericana Sobre Calidad, Eficacia y Cambio En Educación, 14(1), 101-115. https://doi.org/10.15366/reice2016.14.1.006

Surco, D. V. (2018). Gestión Academica y Desempeño Docente, según los estudiantes de una universidad privada en Lima, Perú. Industrial Data, 21(1), 83. https://doi.org/10.15381/idata.v21i1.14914

Tapia, V., \& Tipula, F. (2017). Desempeño docente y creencias pedagógicas del profesor universitario en Universidad Toribio Rodríguez de Mendoza de Amazona . Perú. COMUNI@CCIÓN: Revista de Investigación En Comunicación y Desarrollo, 8(2), 72_ 80. Recuperado de http://www.scielo.org.pe/pdf/comunica/v8n2/a01v8n2.pdf

Torres, E. (2015). Gestión educativa y su relación con la práctica docente en las instituciones educativas emblemáticas de la ciudad de Puno - 2014 - Perú. Comuni@cción,6(1), 5664. Recuperado de http://www.scielo.org.pe/scielo.php?script=sci_arttext\&pid=S2219$71682015000100006 \&$ lang $=\mathrm{pt}$ 